\title{
Translating Geriatric Psychiatry Research into Practice: Coping with Uncertainty
}

\author{
Mark J. Rapoport, MD, FRCPC \\ Department of Psychiatry, Sunnybrook Health Sciences Centre, University of Toronto, Toronto, ON \\ DOI: http://dx.doi.org/10.5770/cgj.15.43
}

As a board member of the Canadian Academy of Geriatric Psychiatry since 2006 and, more importantly, in the course of practicing inpatient geriatric psychiatry in an academic health centre since 2002, I have found a creeping nihilism and cynicism over the last five years among colleagues in general psychiatry and geriatric psychiatry trainees about the treatment of psychiatric disorders in older adults. On further questioning, the pessimism is generally rooted in the anxiety surrounding the publication of large-scale, negative data in this population, and can tend to lead clinicians to a frozen state of uncertainty about how to apply these research results in everyday clinical practice.

A prime example of this is the topic of pharmacological treatment of major depression in late life. Generally speaking, meta-analyses are seen as the highest levels of evidence, and this was certainly the case with the Canadian Coalition for Seniors' Mental Health Guidelines, which were published in $2006 .{ }^{(1)}$ There are a number of meta-analyses of antidepressants in later life that have been published, particularly in recent years. In 2001, Wilson et al. ${ }^{(2)}$ conducted a Cochrane review of randomized controlled trials of antidepressants versus placebo among patients 55 years of age and older. They reported remission rates of $28 \%$ for 245 patients on TCA compared with $17 \%$ for 223 patients on placebo in 10 trials (OR 0.34, 95\% CI 0.21-0.47), and remission rates of $49 \%$ for 365 patients on SSRI versus $25 \%$ for 372 patients on placebo in two clinical trials (OR $0.51,95 \%$ CI $0.36-0.72$ ), corresponding with numbers needed to treat (NNT) of nine for remission with TCA and four for remission with SSRIs.

There were more studies of newer antidepressants to incorporate into the next major meta-analysis on the subject, which was published in 2008. Nelson et al. ${ }^{(3)}$ conducted a meta-analysis of second-generation antidepressants versus placebo among outpatients 60 years of age and older. They pooled 10 studies, and reported a response rate of $44 \%$ among 2,377 patients on second-generation antidepressants compared with $35 \%$ among 1,788 patients on placebo (OR $1.40,95 \%$ CI $1.24-1.57$ ), and remission rates of $34 \%$ of these patients on drug versus $26 \%$ on placebo (OR $1.27,95 \%$ CI 1.12-1.44), corresponding with a calculated NNT of 11 for response and 13 for remission, with better results and less heterogeneity in trials of 10 to 12 weeks in duration. In December of 2011, Tedeschini et al. ${ }^{(4)}$ conducted a meta-analysis of studies of any class of antidepressant versus placebo among patients 55 years of age and older. They found 15 studies, 14 of which were conducted among outpatients, and reported a response rate of $45 \%$ of 2,752 patients on antidepressants compared with $33 \%$ of 2,004 patients on placebo (RR 1.30, 95\% CI 1.15-1.48), corresponding to a NNT of eight. However, when the authors restricted their analysis to the six trials done in patients aged 65 years and older, they reported a non-significant difference in response, with $42 \%$ of 1103 patients on antidepressant versus $39 \%$ of 637 patients on placebo responding (RR $1.28,95 \%$ CI $0.93-1.37$ ). It should also be noted, however, that there was substantial heterogeneity in their analyses. The heterogeneity of results found in both of these recent meta-analyses likely comes from two sources. Firstly, the individual studies incorporated into the metas differed in inclusion and exclusion criteria, drug, dose, number of visits, age, MMSE cut off, and number of sites. Secondly, the sample sizes, confidence intervals, and results of the different studies were quite varied. We should, therefore, be quite cautious in interpreting the pooled response and remission rates, as well as the odds and risk ratios, as the basic assumption of a meta-analysis - that all the studies are asking the same question in a similar manner-was not met. Other concerns about these meta-analyses include inconsistent reporting of conflicts of interest, lack of clarity of the quality of the individual studies, and a focus only on published papers.

The treatment of depression and dementia has become a much more thorny issue over the last year, in the face of some negative evidence that warrants discussion. In 2007, Drs. Sarah Thompson, Nathan Herrmann, Krista Lanctot, and I published a meta-analysis of antidepressants for patients with dementia. ${ }^{(5)}$ At that time, there were only three small studies, but a clear and statistically significant difference was found in favor of antidepressant use, with the number needed to treat of 5.2 for response and 4.5 for remission. A metaanalysis published last summer by Nelson and Devanand ${ }^{(6)}$ incorporated six trials of patients with dementia, including 150 patients on antidepressants and 149 patients on placebo, 
and reported no statistically significant difference between response and remission between the groups. In contrast to the 2007 meta-analysis, however, there was substantial heterogeneity between the studies in the 2011 report. There were two large negative studies on antidepressants in dementia published after the 2007 meta-analysis, one of which was incorporated into the 2011 meta-analysis, and the other was published around the same time. The DIADS-2 study was published in 2010, assessing 67 outpatients who met revised criteria for depression in Alzheimer's disease on sertraline compared with 64 on placebo. ${ }^{(7)}$ There were no differences between the groups at a 12-week endpoint in the Cornell scale for depression in dementia(CSDD). In 2011, the HTA-SADD study found no differences at 13 or 39 weeks in CSDD scores between 107 patients treated with sertraline, 108 patients treated with mirtazapine, and 111 patients treated with placebo, ${ }^{(8)}$ although patients and caregivers in the drug and placebo groups received intensive support, counseling and education sessions which may have diluted the drug-placebo differences, and final doses of sertraline and mirtazapine were significantly below the target doses. In both the HTASADD and the DIADS-2 study, the baseline CSSD scores were less than 15 , on a scale that ranges from 0 to 38 . It is well-known that placebo rates are particularly high in minor depression, and the effectiveness may be higher in those with more severe baseline illness.

Because of those negative studies and increasingly bad press for adverse effects, colleagues are becoming progressively more shy of prescribing SSRIs for depressed elderly. The CMAJ published a retrospective cohort study from Québec last summer examining patients 50 years of age and older who were admitted for an acute myocardial infarction, and discharge home either on ASA or clopidogrel. ${ }^{(9)}$ They reported a $42 \%$ increased risk of bleeding when combining an SSRI with ASA compared with ASA alone (HR 1.42, 95\% CI 1.08-1.8 7), and a 57\% increased risk of bleeding when combining an SSRI with ASA and clopidogrel (HR 1.57, 95\% CI 1.07-2.32). The corresponding number needed to harm for adding SSRI to ASA was 124, and the number needed to harm for adding SSRI to dual antiplatelet therapy was $35 .{ }^{(10)}$ It is important to consider, however, that those treated with an antiplatelet plus an SSRI were more likely to be older, female, have renal or hematological disease, or to be taking other medications, and it is impossible for observational studies to fully correct for these differences. More concerning was a prospective cohort study by Coupland et al. ${ }^{(11)}$ last August examining 60,746 patients aged 65 years and over who had new episodes of depression in UK family practices followed for up to 11 years. The authors reported significant increases in all cause mortality (adjusted HR 1.16, 95\% CI 1.10-1.22), suicide, falls, MIA, stroke, upper G.I. bleeds, seizures, and hyponatremia associated with SSRIs, and they found that these risks were either not present, or substantially less with tricyclic antidepressants. The all cause mortality rates are quite important to note as they correspond with an alarming number needed to harm of $28(1 /[10.61 \%-7.04 \%])$. There are important limitations to this study that the authors acknowledge. Firstly, indication bias would suggest that patients typically treated with SSRIs (i.e., those with depression) may be more likely to die or have adverse medical outcomes than those without depression. This is well borne out in the literature. Secondly, channeling bias would suggest that patients given SSRI antidepressants may be more frail and less likely to be given tricyclic antidepressants, creating an artifactually high adverse event rate in the SSRI group. Thirdly, there may be other residual compounds that the study could not measure.

Given the limitations of the evidence, what can we clinicians do? It is important that we be familiar with the literature, but also with the limitations of literature and the questions that remain open. It is ethically challenging to expose severely ill or suicidal depressed patients to a chance of being entered into a placebo arm of a controlled trial, but these are the patients that we typically treat! There are likely important differences in treatment response between older adults with early-onset vs. late-onset depression, those with and without executive dysfunction or medical illness, particularly cerebrovascular disease, and these aspects of treatment have not been explored in detail in the randomized controlled trials discussed. We must continue to be vigilant in monitoring for adverse effects, but not shy of prescribing antidepressants, particularly when the symptomatology is moderate or severe. We must educate patients, families, and the public about the limitations of the evidence to date, and continue to prescribe according to clinical practice guidelines. Clearly more research is needed, with larger numbers, longer duration, and with patients similar to the ones we treat. Finally, nonspecific therapeutic factors are clearly important, as the non-inert placebo effect shows us, and we should bear this in mind in our clinical practices. Weekly visits with support and family education shouldn't be relegated to an item of lesser importance, and we must advocate for greater availability of psychotherapy services for older adults. Of course, the limitations I've mentioned are not isolated to the topic of major depression in the elderly. There similar controversies in geriatric psychiatry pertaining to antipsychotics in dementia, and the use of cholinesterase inhibitors for Alzheimer's disease and other dementias. There is even less literature in the areas of anxiety disorder, bipolar disorders, substance abuse, delirium, schizophrenia somatoform disorders, or what to do when ECT fails. These are issues that my colleagues and I regularly grapple with.

Last year, the Royal College of Physicians and Surgeons of Canada approved the application, championed by members of the Canadian Academy of Geriatric Psychiatry (CAGP), for an official, college-recognized subspecialty designation of geriatric psychiatrist. The first subspecialty training program in geriatric psychiatry in Canada will start in July 2012 in Toronto, and programs in other academic teaching centres in Canada will most likely follow suit shortly thereafter. Across Canada, existing geriatric psychiatrists will 
need to demonstrate their expertise by completing a Royal college examination in geriatric psychiatry, and the CAGP is helping members consolidate their knowledge in geriatric psychiatry and prepare for the examination by holding an annual two-day review course in geriatric psychiatry taught by leading members in the field. This course will be open to other specialists who may be quite interested in this practical annual clinical and academic update. We have a geriatrician and family physicians actively involved on the planning committee in order to ensure that the course is interesting to non-psychiatrists who care for the elderly as well. The first course will take place immediately following the CAGP annual meeting being held in conjunction with the CCSMH on Sunday, September 23 and Monday, September 24, 2012 in beautiful Banff, Alberta. More information about this course is available at www.cagp.ca. A considerable focus of the course will be further exploration of some of these controversial issues facing us in the field today.

\section{ACKNOWLEDGEMENTS}

I would like to acknowledge Dr. Nathan Herrmann for his scholarly comments on an earlier draft of this manuscript.

\section{CONFLICT OF INTEREST DISCLOSURES}

The author declares that no conflicts of interest exist.

\section{REFERENCES}

1. Tourigny-Rivard M-F, Buchanan D. National guidelines for seniors' mental health: The assessment and treatment of depression. Toronto, ON: Canadian Coalition for Seniors' Mental Health; 2006. Available from: http://www.ccsmh.ca/ en/natlGuidelines/depression.cfm

2. Wilson K, Mottram P, Sivanranthan A, et al. Antidepressant versus placebo for depressed elderly. Cochrane Database Syst Rev. 2001(1):CD000561. Available from: http://onlinelibrary. wiley.com/doi/10.1002/14651858.CD000561/abstract
3. Nelson JC, Delucchi K, Schneider LS. Efficacy of second generation antidepressants in late-life depression: a meta-analysis of the evidence. Am J Geriatr Psychiatry. 2008;16(7):558-67.

4. Tedeschini E, Levkovitz Y, Iovieno N, et al. Efficacy of antidepressants for late-life depression: a meta-analysis and meta-regression of placebo-controlled randomized trials. J Clin Psychiatry. 2011;72(12):1660-68.

5. Thompson S, Herrmann N, Rapoport MJ, et al. Efficacy and safety of antidepressants for treatment of depression in Alzheimer's disease: a metaanalysis. Can J Psychiatry. 2007;52(4):248-55.

6. Nelson JC, Devanand DP. A systematic review and meta-analysis of placebo-controlled antidepressant studies in people with depression and dementia. J Am Geriatr Soc. 2011;59(4):577-85.

7. Rosenberg PB, Drye LT, Martin BK, et al. Sertraline for the treatment of depression in Alzheimer disease. Am J Geriatr Psychiatry. 2010;18(2):136-45.

8. Banerjee S, Hellier J, Dewey M, et al. Sertraline or mirtazapine for depression in dementia (HTA-SADD): a randomised, multicentre, double-blind, placebo-controlled trial. Lancet. 2011;378(9789):403-11.

9. Labos C, Dasgupta K, Nedjar H, et al. Risk of bleeding associated with combined use of selective serotonin reuptake inhibitors and antiplatelet therapy following acute myocardial infarction. CMAJ. 2011;183(16):1835-43.

10. Rapoport MJ, Lawson AJ, Malik K. Research and practice: bleeding and hippocampal atrophy as potential risks of treatment. IPA Bulletin [online]. 2012;29(1).

11. Coupland C, Dhiman P, Morriss R, et al. Antidepressant use and risk of adverse outcomes in older people: population based cohort study. BMJ. 2011;343:d4551.

Correspondence to: Mark Rapoport, MD, FRCPC, Department of Psychiatry, University of Toronto, Sunnybrook Health Sciences Centre, FG37-2075 Bayview Ave, Toronto, ON M4N 3M5.

E-mail: mark.rapoport@sunnybrook.ca 